\title{
Identifying hazards to include in risk analyses
}

\author{
M. Leonhardsen \& O.E. Olsen \\ University of Stavanger
}

\author{
A.S. Nilsen \\ UiT Arctic University of Tromsø
}

\begin{abstract}
A risk analysis should provide decision makers with information regarding relevant hazards. The initiating phase, where the risk analysts identify hazards to be included in the risk analysis, lays the foundation for the rest of the analysis. This phase is, therefore, of great importance. In this paper, we examine how risk analysts in a municipal setting identified potential adverse events and how they chose which ones to analyse in the risk analysis. The municipalities under study had important similarities with respect to exposure to hazards and government regulation. With these similarities as a starting point and studying how the initiating phase took place, the paper focuses on impact regarding the uniformity of adverse events. Looking at events included in the comprehensive risk and vulnerability analyses (CRAs), seems to reveal a predominance of uniformity. This is reasonable given the previously mentioned similarities. It is arguably also a result of many risk analysts using the same sources to retrieve ideas of potential hazards. The latter is alarming when considering risks not listed in these sources, like emergent or local risks.
\end{abstract}

\section{INTRODUCTION}

Communities at different levels of society strive to attain safety and security. To do so, they try to identify hazards and threats that pose a risk. This is a starting point in preparedness for emergency response and risk and vulnerability reduction (Perry \& Lindell 2003). Risk analyses (RAs) are the prominent methods in which risks and vulnerabilities are identified and assessed. They are formal and analytical (Renn 1998; Rausand \& Utne 2009) and used by organizations to prepare for misfortune. They do so by providing decision makers with information about relevant hazards and threats, the likelihood of these adverse events and their potential consequences. RAs enable decision makers to make informed decisions regarding reduction of risks and vulnerabilities (Aven 2011).

The mission of RAs is in other words (Rausand \& Utne 2009):

- To figure out what kind of adverse events might happen

- To figure out the likelihood of the events

- To figure out the consequences of the events

- To describe the risks (Aven 2015)
The bulleted list clearly illustrates that beneficial outcomes of RAs depend on the initiating phase, which is identifying the adverse events of relevance to the RA.

"This is one of the most important steps in the risk analysis. If a hazard source or an adverse event is not detected, it will not be included in the analysis" (Rausand \& Utne 2009, p. 86).

Likewise, Cameron et al. (2017, p. 53) describe the identification of hazards as "the first and most crucial step in any risk assessment". According to Renn (2008), stages of risk assessments vary depending on risk domains and risk sources. Regardless of that, hazard identification is one of three core elements in risk assessment (Renn 2008). Not being able to identify hazards properly can result in accidents or adverse events (Cameron et. al 2017).

Risk analysis has received a lot of academic attention. A December 2017 search for "risk analysis" in the Academic Search Premier database, resulted in approximately 58800 academic articles. Comparatively, searching for "hazard identification" or "identification of hazards" resulted in 1100 and 1250 hits. This paper is a supplement to the studies of this highly important element of risk analysis. 
This paper focuses on the initiating phase of RAs. It presents how risk analysts in 12 municipalities identified and chose hazards to be analysed in greater detail in their RAs. These municipalities had several similarities (they are presented in section 3). With these similarities as a starting point, can we categorise the risk analysts who carried out the work as either copycats at one end of the scale, or explorative analysts at the other?

The main focus, though, is the impact of the approaches used in the initiating phase. This impact is identified and discussed, restricted to uniformity of risks included in the RAs. To be more precise: we have studied the processes when conducting so-called comprehensive risk and vulnerability analyses (CRAs).

Municipalities are exposed to both hazards and threats. The nuances between the two terms are not of importance in our study. So, for convenience, we use "hazard" as a common term for both. Further, we use the terms hazards and adverse events in an interchangeable manner, even though hazards do not necessarily lead to adverse events.

\subsection{The CRAs}

The objective of the Civil Protection Act 2011 and secondary law is to ensure that municipalities safeguard the safety and security of the population (Directorate for Civil Protection 2017). According to these legal requirements, the Norwegian municipalities must have CRAs.

The objective of the Civil Protection Act 2011 and secondary law is to ensure that municipalities safeguard the safety and security of the population (Directorate for Civil Protection 2017). According to these legal requirements, the Norwegian municipalities must have CRAs.

The secondary law lists a few minimum requirements for the CRA. Two of them are of importance for the initiating phase of CRAs (Directorate for Civil Protection 2017):

- First, a CRA must address both existing and future risks in the municipality, as well as external risks of relevance to the municipality.

- Secondly, critical functions in society and critical infrastructure must be addressed. Loss of electricity or water can be examples.

Beyond that, the legal requirements do not specify what kind of adverse events be included in CRAs. Risk analysts in the municipalities must identify the potentially adverse events based on idiosyncratic risks in their communities.

There is a variety of methods for risk analysis (Rausand \& Utne 2009). Analysts in the municipalities are free to choose, but preliminary RAs are the common method in the municipal domain. In preliminary RAs, potential adverse events are identified, then the identified events are analysed separately regarding causes, likelihood and consequences (Aven 2006).

In addition to the requirements in the Civil Protection Act focusing on the risks from a holistic perspective, the municipalities face regulation at the sectorlevel.

\section{THEORETICAL APPROACH}

Several elements are of importance in the initiating phase of RAs. Based on our point of interest, we focus on some theoretical considerations related to the method of risk/hazard analysis, supplemented with some perspectives when suited.

Due to the framework for the paper, elements of importance are excluded, though. For instance, risk perception, i.e. peoples' judgement of hazards (Renn 2008), is not explicitly addressed. Neither is safety culture addressed, even though culture can contribute to focus the attention to some specific hazards, while other hazards are not taken notice of (Pidgeon \& O’Leary, 2000, Pidgeon 1998; Douglas \& Wildavsky 1983).

\subsection{Method and regulation}

A preliminary risk analysis is suited for both major and minor hazards. However, risk analysts might be restricted by a decision that the process of identifying hazards should be limited to regulatory requirements (Baybutt 2014). Such restrictions could, in extreme cases, result in a CRA of rhetorical value, symbolizing control (Clarke 1999), risking that hazards of importance or interest are omitted from the analysis.

\subsection{Imagination}

Cole (2012, p. 12) uses the phrase "broaden the mindset of responders" as an argument for surprise scenarios in exercises. It is also requisite to broaden the mind-set of risk analysts when identifying potentially adverse events.

Imagination and creativity contribute to the identification of scenarios that would otherwise not necessarily have been identified. Hence, imagination and creativity are required, but analysts might lack these characteristics (Camerona et al. 2017). Besides, even if risk analysts are imaginative, it is not a guarantee for identifying all hazards (Baybutt 2014).

A boundary for imagination might be the ontological status of hazards and risks. They are not fixed. Risks can be viewed in different ways; as objective properties or as socially constructed (Aven \& Renn 2010). Risks pre-exist in the former view, and risks can in principle be identified and measured (Lupton 2013 , p. 13). Socially constructed risks, on the other hand, are the product of rhetorical processes (Lupton 2013, p. 46). Potentially this induces discussions or 
interpretations among risk analysts about which hazards to consider in the initiating phase of CRAs.

\subsection{Cognitive biases}

Thinking can be divided into two systems; fast and slow (Kahneman 2011). The fast mode is instinctive and the slow is deliberate. The risk analysis method presupposes deliberate thinking. However, risk analysts are humans. Therefor they are not necessarily as rational as could be expected (Aakvaag 2008).

Cognitive biases are results of heuristics (Kahneman \& Tversky 1982). The biases stem from the unconscious influence on human judgements and decisions (Baybutt 2016). They are deviations from the rationality of thinking (Meissner \& Wulf 2013, p. 802 ). Researchers have found many cognitive biases, e.g. the availability bias, group thinking or the framing bias, to mention a few. We will not go into details in this paper. The point here is that cognitive biases among risk analysts can result in missed hazard scenarios (Baybutt 2016). Therefore the negative effects of cognitive biases need to be addressed. This is very difficult due to the unconscious processes involved (Baybutt 2016). However, knowledge, information and awareness can reduce biases. Another strategy is to use a devil's advocate in the risk analyst team. An appointed devil's advocate can initiate debates that might challenge the mind-set of others (Baybutt 2016). Additionally, scenario planning can alter biases (Meissner \& Wulf 2011).

\subsection{Filtering risks}

There must be a limit to the number of adverse events to analyse in the CRA. It is simply a matter of resources. This implies that the number of identified adverse events in the brainstorming process must be reduced. Rausand \& Utne (2009) argue that hazards where the risks are small, due to low likelihood and/or insignificant consequences, could be filtered here.

Power and interest are also important. Interests can be invested in which adverse events should be emphasised and de-emphasised (Aven 2011; Dekker \& Nyce 2014). This also applies to the brainstorming phase. Being able to handle interests requires the capacity to exercise power. There are several sources of power, e.g. information, expertise, control over agenda and resources (Antonsen 2009).

\subsection{Standardization and uniformity}

Recipes and checklists can be beneficial. They provide advice and save time for risk analysts (Hale \& Swuste 1998). Checklists can also mitigate a lack of imagination among risk analysts (Baybutt 2014).

The purpose of recipes of how to do things is to do the same. Ergo, uniformity is reasonable (Brunsson 2000). However, standardization can cause blindness to possible adverse events unsuited to the recipes (Hale \& Swuste 1998). For instance, Baybutt (2014) holds that elements unlisted in checklists might be left out.

\section{METHODS}

Data were gathered in twelve of nineteen municipalities in a county in Arctic Norway. The main criterion for including municipalities in the study was location. They are all located in the same geographical region, and therefore to a certain extent exposed to the same hazards. Another criterion was time. The Civil Protection Act came into force in 2011. Requirements in the law set a new framework for CRAs. The CRAs and CRA-processes included in the study are from the timespan 2011-2017, ensuring that the municipalities had been subject to the same legal requirements. Their geographical location also meant that they had been subject to the same supervision by the same County Governor. A third criterion was the availability of the informants during the data collection period.

The data was collected via interviews and analyses of the CRAs, a qualitative approach. Twelve semistructured interviews were conducted; one informant per municipality. A question guide with open ended questions was used. The informants all played pivotal roles in the CRA process in their respective municipalities. All of them had participated actively in the process of making the CRAs which this paper focuses on. Hence, they had first-hand knowledge of the process and the choices that were made.

The contents of interviews and CRAs were analysed and compared, so data coherence could be checked.

In a Norwegian context, the municipalities spanned from small to medium population size.

Next, we will present findings from the processes of brainstorming and filtering.

\section{THE BRAINSTORMING PROCESS}

The identification of potential hazards in the municipalities is called the brainstorming process in this paper. The term here refers to a process of creativity, imagination, structure and mapping. Next, we will present the "who's" and the "how's" in this process.

The municipalities had their own unique brainstorming processes. However, there were similarities. Aggregated, the processes either involved

- municipal representatives (M)

- a combination of $\mathrm{M}$ and external representatives (E)

- consultants who involved either M or M+E

In eight of the municipalities, both internal and external representatives participated. 
It is hard to conclude unambiguously in what way the legal requirements and other regulative attempts to influence affected the process of identifying hazards. For some it seems as if regulative involvement broadened the scope of hazards to consider. The internal focus in one of the municipalities was not motivated by legal compliance. The intention was to heighten organizational competence in this area of municipal responsibility. A contrast is the municipality with the lowest involvement in this study. Here the primary objective was a "good enough" CRA.

A devil's advocate formally appointed to challenge assumptions or stimulate ideas was not used. Ascribing such a formal role to a participant in the processes seems to be unfamiliar to the risk analysts. However, in two of the municipalities, the risk analysts responsible for the local processes deliberately sought counter-arguments. They were self-appointed informal devil's advocates.

A common trait for the municipalities is that they based identification of hazards on a combination of information sources. In some cases, their imagination was not sufficient, and other sources provided valuable inspiration and ideas. Nobody referred to checklists etc. as means to save time or resources.

In addition to their own previous municipal CRAs, most analysts used regional or national sources to assist them when identifying hazards: typically, national and regional RAs and a government guideline for CRAs. The analyses and the guideline served as checklists. At the local level, other municipal CRAs were sources of information too, but to a lesser extent than for instance the regional RA.

Another influence was the urge from national and regional government to take specific adverse events into consideration, for instance deliberate adverse events in schools etc. (threats, use of weapons), and quite recently, arrival of refugees in large numbers.

Media-coverage was also a source of information and inspiration to some risk analysts.

The informants also referred to a recently established regional arena for risk analysts. Here the analysts could exchange ideas. For instance, the hazard related to cruise tourism had been addressed by one of the municipalities. This was also relevant for some of the other municipalities. The influence from this arena will probably be apparent in future CRAs.

Arguably, these sources facilitate uniformity if not reflected on.

Interestingly, in two of the municipalities, representatives from residents were invited to participate in the process, potentially providing a local focus. Representatives from the municipality and external actors who had been invited to contribute could, of course, also add a new perspective.

The analysts were asked about the usefulness of external information sources and potential negative effects. The majority found such sources very helpful, providing ideas and serving as some sort of quality control as to the content of CRAs. The potential negative effects seem to be eliminated by the usefulness of such sources.

\section{THE FILTERING PROCESS}

After having identified potential hazards, the municipalities chose which hazards to include in the CRA. In this paper, this process is called filtering. Some of them had identified many potential adverse events, others had few. Most of the municipalities structured the identified events by merging related events, thus reducing the number of events. In addition, the approach of filtering out small risks was applied in several municipalities.

One of the municipalities in fact included all of the identified hazards in the CRA as they were, without filtering.

All in all, the filtering processes passed without much controversies, according to the risk analysts. Issues for debate were the severity of hazards, not their ontological status. The participants in the process came to an agreement. External actors without representation in the CRA work, such as representatives from local industry, showed no interest in trying to influence this, or other, processes. This lack of interest is interesting per se, but beyond the scope of this paper.

\section{IMPACT}

Looking at the type of risks included in the CRVs, there is a high degree of uniformity regarding events that are mandatory to address; e.g. critical infrastructure.

However, the analysts have not analysed all types of critical infrastructure. They have chosen the ones relevant to them. Electricity and electronic communication are the focus of attention, followed by water supply. Transportation is also addressed in some of the CRAs. Here, local circumstances are obviously of importance. E.g. municipalities with only one main road are more vulnerable than those with several.

Pandemics, nuclear accidents and extreme weather, the transboundary risks, are also included to a high degree in the CRAs. Fires, accidents, emissions or spills of dangerous substances are also addressed to a high degree. These are events of relevance to all municipalities, regardless of location. However, the detailing and the objects at risk vary from one CRA to the other. Municipalities with a coastline have analysed accidents at sea, for example.

Several of the above-mentioned hazards are regulated in sector legislation: e.g. water-supply, fires and nuclear accidents.

There is also high uniformity at an aggregated level regarding deliberate adverse events. They are 
included in the CRAs. The types of adverse events differ, though. Events like threats and "minor" violence are addressed by almost everybody. Terror, a disastrous event, is covered in fewer CRAs. Here, analysts have varied between the events, most likely based on their assessment of relevance to their municipality. Only two CRAs include Cyber-attack. This type of hazard has not been on the public agenda for very long, and the two CRAs have recently been revised.

In addition, the CRAs encompass a few adverse events of a strictly local character: flooding and the breaking of dikes.

Finally, a few of the CRAs contain unique adverse events; such as substance abuse among municipal employees, animal diseases, violence and sexual abuse against children and breaches in information security.

Identifying adverse events is a challenging task, taking uncertainty about what the future holds into consideration. In two of the municipalities this was addressed by including "the unknown event".

\section{DISCUSSION AND CONCLUSION}

\subsection{Discussion}

The preliminary risk analysis method and the legal requirements per se are neutral regarding the number of people involved. The variety among the studied municipalities is vast with respect to involvement of personnel. At one end of the continuum only the consultant and a single municipal employee took part in the process. Here the rhetorical value of the CRA was the primary objective, i.e. having a CRA in compliance with regulations (Clarke 1999). At the other end of the continuum a bottom-up approach was applied with all municipal departments being mobilized. The variety of involvement does not seem to have a bearing on the number of adverse events included in the CRAs.

If other parameters were considered, like how well founded the CRAs are in the municipality, or reduction of risk, then the verdict regarding choice of processes might shift. Important hazards might be missing in the CRA (Clarke 1999).

There is a predominance of uniformity in the events that were included in the CRAs. Obviously, this can be ascribed to legal requirements and that the municipalities face the same hazards to a relatively large degree. In addition, the formal framework for CRAs confers some uniformity. The brainstorming processes also induced uniformity, because many risk analysts in the study used the same sources to retrieve ideas of potential hazards. With recipes and a framework like this, some uniformity is reasonable (Brunsson 2000)

Still, we would argue that this is alarming with regard to hazards not listed in these sources, like emergent, novel or local hazards. They might be left out, as implied by Baybutt (2014) and Hale \& Swuste (1998). For instance, fish diseases were not included in one of the often-used sources, the County-RA. This hazard might be of relevance in several municipalities because fishery is a prominent part of the industrial base in these communities. Only one CRA included this hazard. Using sources to retrieve ideas, the risk analyst might miss out hazards if the process resembles copying and has a lack of imagination (Cameron et al. 2017; Baybutt 2014).

Most of the processes took place without major disagreement. A devil's advocate was not used in most of the municipalities. Hence, the processes lacked a participant who systematically could have challenged the mind-set of others (Baybutt 2016). Given the uncertainty about future adverse events and local susceptibility, the processes could have benefited from critical voices challenging both the premises for analysing risks, i.e. the formal framework for CRAs, and the local processes.

This could perhaps have provided more diversity in novel or unique adverse events. However, diversity is not an objective per se. The CRAs were in fact diverse in having variations within types of hazards. For instance, some included car accidents, others included bus accidents.

Having argued in this paper that uniformity can be alarming, a final reflection should be added regarding crisis management. Even if all hazards have not been identified and analysed, in crisis management many of the same features occur regardless of the hazard involved (Nilsen 2017). Therefore, uniformity need not be too serious in that respect. The disadvantage of uniformity is the decreased possibility of reducing or eliminating unknown hazards in advance, making crisis management redundant.

\subsection{Conclusion}

The initiating phase of any risk analysis, where adverse events are identified and filtered, is very important. This paper has addressed how risk analysts in 12 municipalities have carried out this phase and the impact of uniformity of adverse events analysed in municipal CRAs. The main conclusion is that there is a predominance of uniformity in CRA-events, as could be expected due to the circumstances and the brainstorming-processes.

Diversity is not an objective per se. However, the chance of identifying the unknown adverse event is lessened by copycats. That is alarming, and awareness needs to be raised.

\section{REFERENCES}

Antonsen, S. 2009. Safety culture and the issue of power. Safety Science, 47: 183-191 
Aven, T. 2015. Risikostyring [Risk management. Own translation]. $2^{\text {nd }}$ ed. Oslo: Universitetsforlaget.

Aven, T. 2011. Selective critique of risk assessments with recommendations for improving methods and practice. Reliability Engineering and System Safety, 96:509-514

Aven, T. 2006. Pålitelighets-og risikoanalyse [Reliability- and risk analysis. Own translation]. $4^{\text {nd }}$ ed. Oslo: Universitetsforlaget

Aven, T. \& Renn, O. 2010. Risk Management and Governance. Concepts, Guidelines and Applications. Berlin: Springer

Aakvaag, G. 2008. Moderne sosiologisk teori [Modern sociological theory. Own translation]. Oslo: Abstrakt forlag AS

Baybutt, P. 2016. Cognitive biases in process hazard analysis. Journal of Loss Prevention in the Process Industries, 43:372-377

Baybutt, P. 2014. A critique of the Hazard and Operability (HAZOP) Study. Journal of Loss Prevention in the Process Industries, 33:52-58

Brunsson, N. 2000. Standardization and Uniformity. In Brunsson N. and Jacobsson, B. eds. A world of standards. New York: Oxford University Press, p. 138-150.

Cameron, I., Mannan, S., Németh E., Park, S, Pasman H., Rogers W. \& Seligmann B. 2017. Process hazard analysis, hazard identification and scenario definition: Are the conventional tools sufficient, or should and can we do much better? Process Safety and Environmental Protection, 10:53-70

Clarke, L.B., 1999. Mission improbable: using fantasy documents to tame disaster. Chicago: University of Chicago Press

Cole, L.A. 2012. Preparedness, Uncertainty, and Terror Medicine. In L.A. Cole \& N.D. Connell (eds.), Local Planning for Terror and Disaster. From Bioterrorism to Earthquakes, p. 3-15. New Jersey: Wiley

Dekker, S.W.A \& Nyce, J.M. 2014. There is safety in power, or power in safety. Safety Science 67: 44-49

Directorate for Civil Protection (DSB). 2017. Veileder til helhetlig risiko- og sårbarhetsanalyse i kommunen [Guideline for comprehensive risk and vulnerability analysis in the municipality. Own translation]. Tønsberg: Direktoratet for samfunnssikkerhet og beredskap

Douglas, M. \& Wildavsky, A. 1983. Risk and Culture. An Essay on the Selection of Technological and Environmental Dangers. Berkley: University of California

Hale, A.R \& Swuste, P. 1998. Safety rules: procedural freedom or action constraint? Safety Science, 29(3): 163-177

Kahneman, D. 2012. Tenke, fort og langsomt. [Thinking, fast and slow]. Oslo: Pax forlag

Lupton, D. 213. Risk. 2nd ed. London and New York: Routledge

Nilsen, A.S. 2017. What challenges can municipalities experience in crisis management? In Cepin M. and Bris, R.(eds.). Safety and Reliability. Theory and Applications. London: Taylor \&Francis Group, p. 1747-1754

Meissner, P. \& Wulf, T. 2013. Cognitive benefits of scenario planning: Its impact on biases and decision quality. Technological Forecasting and Social Change, 80(4): 801-814

Perry, R.W. \& Lindell, M.K. 2003. Preparedness for Emergency Response: Guidelines for the Emergency Planning Process. Disasters. 27(4): 336-350

Pidgeon, N. \& O'Leary, M. 2000. Man-made disasters: why technology and organizations (sometimes) fail. Safety Science, 34:15-30.

Pidgeon, N. 1998. Safety culture: Key theoretical issues. Work \& Stress, 12(3): 202-216

Renn, O. 2008. Risk Governance. Coping with uncertainty in a Complex world. London/New York: Earthscan

Rausand, M. \& Utne, U.B. 2009. Risikoanalyse - teori og metode [Risk analysis - theory and methods. Own translation]. Trondheim: Tapir akademiske forlag

Tversky, A. \& Kahneman, D. 1974. Judgment under uncertainty: heuristics and biases. Science, 185, p. 1124-1131 